\title{
Treatment of postmenopausal osteoporosis, patient perspectives - focus on once yearly zoledronic acid
}

This article was published in the following Dove Press journal:

Patient Preference and Adherence

7 July 2009

Number of times this article has been viewed

\section{Raj Carmona \\ Rick Adachi}

Division of Rheumatology Department of Medicine, McMaster University, Hamilton, Ontario, Canada
Correspondence: Raj Carmona 187 Hughson St South, Hamilton, Ontario, Canada, L8N 2B6

Tel + I 905-528-I I 23

Email rajcarmona@hotmail.com

\begin{abstract}
Oral bisphosphonates are of proven efficacy in preventing fractures in postmenopausal osteoporosis. However, poor adherence limits their real-world efficacy and clinical utility. Zoledronic acid (ZOL) is a potent bisphosphonate administered by annual intravenous infusion, effectively ensuring adherence to therapy over the following year. According to available data, $66 \%$ to $79 \%$ of patients have expressed a preference for ZOL over oral bisphosphonates. This is likely to lead to enhanced clinical outcomes, although long-term (repeat annual) adherence is currently unknown. ZOL is of proven efficacy, with hip fracture reduction of $41 \%$ and morphometric vertebral fracture reduction of $70 \%$ over 3 years in the HORIZON PFT trial. It has demonstrated a good side-effect profile with postinfusion flu-like symptoms being the most common. Additionally, it has been associated with decreased mortality in patients following surgery for hip fracture. There is no clear association between exposure and the rate of serious or nonserious atrial fibrillation. We review adherence to oral bisphosphonates, and the pharmacokinetics, efficacy, safety, and patient preference for ZOL.
\end{abstract}

Keywords: zoledronic acid, bisphosphonate, osteoporosis, fractures

\section{Introduction}

Postmenopausal osteoporosis (PMO) is a chronic condition characterized by decreased bone mass and increased risk of fragility fracture. Such fractures lead to pain, disability, impaired quality of life, increased risk of death, and annual health-care costs in the United States of at least US\$19 billion (2005 figures). ${ }^{1}$ The average woman and man older than 50 years have a $50 \%$ and $25 \%$ risk of osteoporotic fracture in their remaining lifetime respectively. ${ }^{1}$ Worldwide, there were an estimated 1.66 million hip fractures in $1990^{2}-\mathrm{a}$ number that is likely increasing with an aging population. Additionally, hip fractures cause more total days of disability than any other clinical osteoporotic fracture. ${ }^{3}$ Compounding this, $20 \%$ to $24 \%$ of patients die within the first year following hip fracture, and only half of survivors regain full independence. ${ }^{4}$ Therefore, timely diagnosis, provision of effective therapy and adherence to therapy are of paramount importance.

Like other chronic diseases though, particularly asymptomatic ones, compliance and persistence (collectively called "adherence") with PMO therapy are abysmal. In one longitudinal cohort study, $68 \%$ of patients on a daily bisphosphonate regimen and $56 \%$ on a weekly regimen had discontinued therapy at 1 year. ${ }^{5}$ Data from a United Kingdom database showed that at 1 year $42 \%$ had discontinued bisphosphonates, and $76 \%$ had done so by 5 years. ${ }^{6}$ In another study, ${ }^{7} 47 \%$ of patients at 6 months and $54 \%$ at 1 year had suboptimal adherence (medication possession ratio $[\mathrm{MPR}]<80 \%$ ), with similar trends in other studies. 
Unfortunately, the best drug will not work in the patient who does not use it. Although oral bisphosphonates have confirmed efficacy in reducing fragility fractures, poor adherence limits their clinical utility and negatively impacts on outcomes. Data from two US claims databases showed that adherence (MPR $>80 \%$ ) reduced nonvertebral fracture risk by $20 \%$, and hip fracture risk by $45 \%$ ( $P<0.001$ for both). ${ }^{8}$ Adherence levels $<50 \%$ did not have any effect on fracture risk, but risk decreased as adherence increased above $50 \%$, with a steep improvement between $75 \%$ and $100 \%$. The aforementioned UK study showed no fracture risk reduction in those receiving less than 6 months of therapy. ${ }^{6}$ These data suggest maximal benefit with maximum adherence. They also suggest that even with some degree of drug use, suboptimal adherence can lead to a total loss of any benefit.

Although several studies of adherence to PMO therapy have found significant differences between adherent and nonadherent patients, attempts to develop a predictive model have been unsuccessful. ${ }^{9}$ Nonadherence to oral bisphosphonates is partly due to the frequency of dosing, inconvenience of administration requiring a fasting state pre-/postingestion, and gastrointestinal side-effects. ${ }^{10}$ Less frequent dosing with weekly and monthly oral regimens are generally preferred by patients over daily dosing, but are still associated with suboptimal adherence. ${ }^{11}$ In one study, younger age, female sex, fewer comorbid conditions, fewer PMO medication, and bone mineral density (BMD) testing each independently predicted compliance, but models accounted for only $6 \%$ of the variation seen. ${ }^{12}$ Surprisingly, associations of prior fracture with adherence have been inconsistent.

Intravenous (IV) zoledronic acid (ZOL), $5 \mathrm{mg}$ administered annually, provides the greatest extended dosing interval, and reduces concerns about oral administration, gastrointestinal intolerance and bioavailability. In two separate trials of outpatients comparing weekly oral alendronate to annual ZOL, 66\% and 79\% preferred the latter. ${ }^{13,14}$ While not all patients may comply with ZOL, and little is known about long-term adherence, it does essentially provide $100 \%$ adherence in the year following administration in those who choose to receive it.

\section{Pharmacology and kinetics}

Preclinical data indicate that ZOL, a nitrogen-containing bisphosphonate, is one of the most potent bisphosphonates available. It has a higher binding affinity for hydroxyapatite and is a more potent inhibitor of farnesyl disphosphonate synthase and bone resorption than other bisphosphonates. ${ }^{15}$ ZOL has demonstrated inhibition of bone resorption in vivo at doses of $0.072 \mu \mathrm{g} / \mathrm{kg} .{ }^{15}$ Dynamic bone histomorphometry has been evaluated in 93 patients with PMO. Results showed normal quality bone, preservation of trabecular architecture, with no evidence of impaired remodelling or mineralization defects. ${ }^{16}$

Pharmacokinetic data are not available for PMO. When assessed in 64 cancer patients, however, postinfusion decline in ZOL plasma concentrations showed a triphasic process, with a rapid decrease in the first 24 hours, and prolonged terminal elimination phase. ${ }^{16}$ An average of $39 \pm 16 \%( \pm \mathrm{SD})$ was excreted in urine within the first 24 hours, with only trace amounts after 48 hours. The balance of drug, presumably bound to bone, is slowly released back into the systemic circulation, giving rise to the observed prolonged low plasma concentrations. ZOL is not recommended for patients with severe renal impairment $(\mathrm{CrCl}<30 \mathrm{~mL} / \mathrm{min})$ due to lack of experience in this population. No dosage adjustment is required with a $\mathrm{CrCl}>30 \mathrm{~mL} / \mathrm{min} .{ }^{16} \mathrm{ZOL}$ is not metabolized in humans, and exhibited little or no capacity as a directacting and/or irreversible metabolism-dependent inhibitor of P450 enzymes. ${ }^{16}$ It is therefore unlikely to affect compounds metabolized by this enzymatic system.

\section{Efficacy studies}

The use of biomarkers is one method of assessing the impact of antiresorptive therapy, with lower levels demonstrating decreased resorption and bone turnover. Such biomarkers

\section{Table I Key points of zoledronic acid}

\section{Fracture prevention with oral bisphosphonates in PMO is limited by nonadherence, partly due to $\mathrm{Gl}$ intolerance, frequency of administration and inconvenience of dosing. \\ 2. For $P M O, Z O L$ is administered annually as a $5 \mathrm{mg}$ infusion. \\ 3. Efficacy of ZOL in PMO has been confirmed in the HORIZON PFT trial: $41 \%$ reduction in hip fractures, $70 \%$ reduction in morphometric vertebral fractures.}

4. ZOL is the only bisphosphonate that is associated with decreased mortality (post-hoc analysis) following hip fracture (HORIZON RFT).

5. ZOL has a good safety profile. The most common side-effects are postinfusion flu-like symptoms, but these significantly diminish with successive infusions.

6. $66 \%$ to $79 \%$ of patients have expressed a preference for ZOL, including those with postinfusion symptoms.

7. When administered, IV ZOL effectively guarantees adherence for 12 months following infusion, and will therefore likely decrease fracture rates in the "real-world" setting.

8. Cost-effectiveness and head-to-head comparison with oral bisphosphonates are needed to fully elucidate the role of ZOL in PMO.

Abbreviations: PMO, postmenopausal osteoporosis; ZOL, zoledronic acid; HORIZON, Health Outcomes and Reduced Incidences with ZOL Once Yearly trial; PFT, Pivotal Fracture Trial; RFT, Recurrent Fracture Trial; IV, intravenous. 
include urine $\mathrm{N}$-telopeptide of type I collagen (NTX), serum $\beta$-C-telopeptide of type I collagen ( $\beta$-CTX), and bone-specific alkaline phosphatise. When compared to weekly alendronate in a 24-week trial, ${ }^{13}$ ZOL showed faster reduction in mean urine NTX at 1 week, and greater reduction in NTX and $\beta$-CTX and at all follow-up points, implying faster and greater reduction in bone turnover.

The Health Outcomes and Reduced Incidences with ZOL Once Yearly (HORIZON) Pivotal Fracture Trial (PFT) ${ }^{17}$ was an international placebo-controlled randomized double-blind trial $(\mathrm{n}=7736)$. It assessed the efficacy of $5 \mathrm{mg}$ IV ZOL given annually for 3 years in reducing new vertebral and hip fractures. The patients were women aged 65 to 89 years with PMO. All patients received daily calcium (1000 to $1500 \mathrm{mg}$ ) and vitamin D (400 to 1200 IU). At 3 years, ZOL significantly reduced the relative risk of morphometric vertebral fracture by $70 \%(3.3 \%$ vs $10.9 \%, P<0.001)$, and hip fracture by $41 \%$ (1.4\% vs $2.5 \%, P=0.002)$. ZOL also significantly reduced the incidence of clinical vertebral fracture by $77 \%(0.5 \%$ vs $2.6 \%, P<0.001)$, nonvertebral fractures by $25 \%$ (8.0\% vs $10.7 \%, P<0.001)$, and all clinical fractures by $33 \%(8.4 \%$ vs $12.8 \%, P<0.001)$. BMD at the total hip, lumbar spine and femoral neck were increased significantly, by $6.0 \%, 6.7 \%$, and $5.1 \%$, respectively, compared with placebo $(P<0.001$ for all comparisons).

The subsequent HORIZON Recurrent Fracture Trial (RFT) ${ }^{18}$ examined the efficacy of ZOL in 2127 patients $(24 \%$ men, $76 \%$ women) who had undergone surgical repair of a low-trauma hip fracture. Patients must have been unable or unwilling to take oral bisphosphonates and were randomized within 90 days of fracture to ZOL $(n=1065)$ or placebo $(\mathrm{n}=1062)$. Median follow-up was 1.9 years. There were significant relative risk reductions in new clinical fractures (35\%), clinical vertebral fractures $(46 \%)$, and nonvertebral fractures $(27 \%)$, and a nonsignificant $30 \%$ reduction in hip fractures $(P=0.18)$.

Of note, there have been no head-to-head clinical trials to date comparing the efficacy of different bisphosphonates with fracture as the primary endpoint.

\section{Safety and tolerability}

ZOL was well tolerated in the HORIZON PFT trial. ${ }^{17}$ The most common adverse events were postdose symptoms: pyrexia, myalgia, headache, arthralgia, or influenza-like symptoms. Any or a combination of these occurred primarily following the first infusion (31.6\%). This progressively decreased with subsequent infusions to affect only $2.8 \%$ after the third infusion. Such symptoms can be mitigated with acetaminophen or ibuprofen before and after the first administration. ${ }^{18}$

As in some studies of other bisphosphonates, arrhythmias in the HORIZON PFT were significantly higher in the ZOL group (6.9\% vs 5.3\% placebo, $P=0.003)$, including 50 patients with serious atrial fibrillation (AF) $(1.3 \%$ vs $0.5 \%$ placebo, $P<0.0001)$. Of these 50 patients, however, 47 developed AF more than 30 days postinfusion. It must be noted that in patients who underwent electrocardiography, there was no difference in AF detection (2.1\% vs $2.8 \%$ placebo). There was no difference in the occurrence of stroke ( $2.8 \%$ in both groups). ZOL did not increase cardiovascular mortality - there was no significant difference in death due to stroke $(0.5 \% \mathrm{ZOL}$ vs $0.3 \%$ placebo, $P=0.15)$, or cardiovascular causes $(1.0 \% \mathrm{ZOL}$ vs $0.9 \%$ placebo, $P=0.55)$. In the HORIZON RFT, there was no increase in the risk of AF $(1.1 \%$ in the ZOL group vs $1.3 \%$ in the placebo group, $P=0.84$ ). It is noteworthy that this study was conducted in the postoperative period, a time that usually confers increased risk of arrhythmias. The FDA continues to monitor data regarding atrial fibrillation, and has stated that "across all studies, no clear association between overall bisphosphonate exposure and the rate of serious or nonserious atrial fibrillation was observed. Increasing dose or duration of bisphosphonate therapy was also not associated with an increased rate of atrial fibrillation" ${ }^{19}$ Consequently, the FDA has advised that healthcare professionals should not alter their prescribing patterns for bisphosphonates, and patients should not discontinue therapy for this concern.

As mentioned, ZOL is not recommended in those with creatinine clearance $<30 \mathrm{~mL} / \mathrm{min}$, and patients with $\mathrm{CrCl}<30 \mathrm{~mL} / \mathrm{min}$ were excluded from the HORIZON trial. A greater proportion of patients in the ZOL group had a transient increase in serum creatinine of more than $0.5 \mathrm{mg} / \mathrm{dL}$ at 9 to 11 days postinfusion $(1.2 \%$ vs $0.4 \%$ placebo, $P=0.001)$. By 30 days, however, $85 \%$ had returned to within $0.5 \mathrm{mg}$ of preinfusion values, and the remainder had reverted to this range by the next annual follow-up.

In the HORIZON-RFT, ${ }^{18} \mathrm{ZOL}$ was associated with a $28 \%$ lower mortality risk compared to placebo infusions $(P=0.01)$. This was the first time such a benefit was demonstrated for a PMO medication. The rates of renal and cardiovascular adverse events were similar to placebo.

All bisphosphonates carry the same FDA-mandated class warning regarding osteonecrosis of the jaw (ONJ), defined as oral exposed bone lesions not healing after $>8$ weeks from identification. ${ }^{20}$ There were no spontaneous reports of ONJ in the HORIZON-PFT or HORIZON-RFT trials. 
In HORIZON-PFT, one patient in each group had delayed wound healing classified as potential ONJ, but both cases resolved with antibiotics and limited debridement.

\section{Patient preference}

One can expect patient preference for therapies with extended dosing intervals, as demonstrated in several studies comparing daily, weekly and monthly oral bisphosphonates. In a recent double-blind, randomized, placebo-controlled trial assessing the safety and biomarker changes of weekly oral alendronate vs annual ZOL, 79\% of patients preferred an annual infusion. ${ }^{14}$ Similarly, Saag et al reported that a majority of patients $(66 \%)$, including those experiencing flu-like symptoms, expressed a preference for annual ZOL versus weekly alendronate. ${ }^{13}$

An earlier study showed that when given the hypothetical choice of PMO therapies, 65\% of treatment-naïve patients with BMD-confirmed osteoporosis chose to receive an annual IV bisphosphonate infusion. ${ }^{21}$ Patients with a poorer perceived health status, high perceived risk of future fracture, and those preferring to manage their health without doctors, were more likely to choose an annual IV infusion. Patients unable to tolerate or properly take oral bisphosphonates were excluded from this study, to avoid bias towards IV therapy. Among those who were already on bisphosphonates, only a preference for prescription drugs predicted preference for oral therapy. The added benefit of recombinant human parathyroid hormone was not sufficient to overcome the dislike of daily subcutaneous injections.

Despite these studies demonstrating preference for annual ZOL, it is not known whether IV bisphosphonates improve long-term adherence beyond the initial infusion. The rate of return for a second infusion has not been established.

\section{Conclusions}

Despite proven efficacy, the clinical benefit of oral bisphosphonates is significantly limited by nonadherence to therapy. ZOL, by virtue of its intravenous administration, extended dosing interval, improved bioavailability, proven efficacy, and minimal side-effects, is an excellent option. Admittedly, there are no data on safety or sustained efficacy beyond 3 years of therapy (the follow-up period in HORIZON). At this time, though, there is no reason to suspect that these would change. It is also not known whether the current annual $5 \mathrm{mg}$ IV regimen provides optimal efficacy.

As described, patients have expressed a strong preference for IV bisphosphonates in several studies. The effectively guaranteed 12 months of adherence following infusion distinguishes ZOL from other bisphosphonates, and will likely lead to decreased vertebral and nonvertebral fractures in the "real-world" setting. This will not only benefit PMO patients directly, but also lead to decreased fracture-related healthcare costs. Proper health-economic analysis will be needed to clarify this cost-effectiveness issue, however.

We believe that ZOL should be the therapy of choice in PMO patients for whom bisphosphonates are indicated, and who have a malabsorption syndrome, are intolerant of oral therapy, or are nonadherent (suspected or confirmed). Additional head-to-head comparisons with oral bisphosphonates, with fracture outcomes, are needed to fully elucidate the role of $\mathrm{ZOL}$ in the treatment of PMO.

\section{Disclosures}

The authors declare no conflicts of interest.

\section{References}

1. National Osteoporosis Foundation. Disease Statistics. Available online at http://www.nof.org/osteoporosis/diseasefacts.htm. Accessed April 15, 2009

2. Cooper C, Campion G, Melton L. Hip fracture in the elderly: A worldwide projection. Osteoporosis Int. 1992;48:285-289.

3. Fink H, Ensrud K, Nelson D, Kerani R, Schreiner P, Zhao Y, et al. Disability after clinical fracture in postmenopausal women with low bone density: the fracture intervention trial (FIT). Osteoporosis Int. 2003; 14:69-76.

4. Mazanec D. Osteoporosis screening: time to take responsibility. Arch Intern Med. 2004;164:1047-1048.

5. Recker R, GallagherR, MacCosbe P. Effect of dosing frequency on bisphosphonates medication adherence in a large longitudinal cohort of women. Mayo Clin Proc. 2005;80:856-861.

6. Gallagher A, Rietbrock S, Olson M, Van Staa T. Fracture outcomes related to persistence and compliance with oral bisphosphonates. J Bone Miner Res. 2008;23:1569-1575.

7. Huybrechts K, Ishak K, Caro J. Assessment of compliance with osteoporosis treatment and its consequences in a managed care population. Bone. 2006;38:922-928.

8. Siris E, Harris S, Rosen C, Barr C, Arvesen J, Abbott T, et al. Adherence to bisphosphonate therapy and fracture rates in osteoporotic women: relationship to vertebral and nonvertebral fractures from 2 US claims databases. Mayo Clin Proc. 2006;81:1013-1022.

9. Badamgarav E, Fitzpatrick L. A new look at osteoporosis outcomes: the influence of treatment, compliance, persistence, and adherence. Mayo Clin Proc. 2006;81:1009-1012.

10. Cramer J, Gold D, Silverman S, Lewiecki E. A systematic review of persistence and compliance with bisphosphonates for osteoporosis. Osteoporosis Int. 2007;18:1023-1031.

11. Reginster J, Rabenda V, Neuprez A. Adherence, patient preference and dosing frequency: understanding the relationship. Bone. 2006; 38(4 Suppl 1):S2-S6.

12. Solomon D, Avorn J, Katz J, Finkelstein J, Arnold M, Polinski J, et al. Compliance with osteoporosis medications. Arch Intern Med. 2005;165:2414-1419.

13. Saag K, Lindsay R, Kriegman A, Beamer E, Zhou W. A single Zoledronic acid infusion reduces bone resorption markers more rapidly than weekly oral alendronate in postmenopausal women with low bone mineral density. Bone. 2007;40:1238-1243. 
14. McClung M, Recker R, Miller P, Fiske D, Minkoff J, Kriegman A, et al. Intravenous Zoledronic acid $5 \mathrm{mg}$ in the treatment of postmenopausal women with low bone density previously treated with alendronate. Bone. 2007;41:122-128.

15. Green J, Muller K, Jaeggi K. Preclinical pharmacology of CGP 42'446, a new, potent, heterocyclic bisphosphonate compound. J Bone Miner Res. 1994;9:745-751.

16. Novartis Pharmaceuticals Canada Inc. Aclasta product monograph. June 2005 (revised March 2009).

17. Black D, Delmas P, Eastell R, Reid I, Boonen S, Cauley J, et al. Onceyearly zoledronic acid for treatment of postmenopausal osteoporosis. N Engl J Med. 2007;356:1809-1822.

18. Lyles K, Colon-Emeric C, Magaziner J, Adachi J, Pieper C, Mautalen C, et al. Zoledronic acid and clinical fractures and mortality after hip fracture. N Eng J Med. 2007;357:1799-1809.
19. U.S. Food and Drug Administration. Update of safety review to the October 1, 2007 early communication about the ongoing safety review of bisphosphonates. Nov 12, 2008. Available online at http:/www. fda.gov/cder/drug/early_comm/bisphosphonates_update_200811.htm. Accessed April 15, 2009.

20. Khosla S, Burr D, Cauley J, Dempster D, Ebeling P, Felsenberg D, et al. Bisphosphonate-associated osteonecrosis of the jaw: report of a task forceof the American Society for Bone and Mineral Research. J Bone Miner Res. 2007;22:1479-1491.

21. Fraenkel L, Gulanski B, Wittink D. Patient treatment preferences for osteoporosis. Arth Rheum. 2006;55:729-735.

Patient Preference and Adherence

\section{Publish your work in this journal}

Patient Preference and Adherence is an international, peer-reviewed, open access journal that focusing on the growing importance of patient preference and adherence throughout the therapeutic continuum. Patient satisfaction, acceptability, quality of life, compliance, persistence and their role in developing new therapeutic modalities and compounds to optimize clinical outcomes for existing disease states are major areas of interest. This journal has been accepted for indexing on PubMed Central. The manuscript management system is completely online and includes a very quick and fair peer-review system. Visit http://www.dovepress.com/ testimonials.php to read real quotes from published authors.

Submit your manuscript here: http://www.dovepress.com/patient-preference-and-adherence-journal 